\title{
Endoscopic Submucosal Dissection for Early Gastric Cancer
}

\author{
Kouichi Nonaka*, Hiroto Kita \\ Department of Gastroenterology, Saitama Medical University International Medical Center, Saitama, Japan. \\ Email: *nonaka513@gmail.com
}

Received November $15^{\text {th }}, 2012$; revised December $17^{\text {th }}, 2012$; accepted December $28^{\text {th }}, 2012$

\begin{abstract}
The field of endoscopy has progressed markedly and become widespread in recent years, and the role of minimally invasive endoscopic treatment has become increasingly more important with the increase in the number of patients in whom gastric cancer is detected at an early stage. In addition, the characteristics of early gastric cancer, which can be curably treated by mucosal resection alone just as by surgical cancer resection, were clarified, and endoscopic submucosal dissection (ESD) was developed as a highly curable, minimally invasive treatment, that is gaining popularity. In this paper, we describe the technical details and complications of ESD for early gastric cancer, including their management.
\end{abstract}

Keywords: Gastric Cancer; ESD; Endoscopic Submucosal Dissection

\section{Introduction}

More than 20 years have passed since the introduction of endoscopic mucosal resection (EMR) to the treatment of digestive tract tumors, and endoscopic treatment is now widely performed for early digestive tract cancers including stomach as well as esophageal and colon cancers [1-4]. Endoscopic submucosal dissection (ESD) was reported as treatment for early-stage gastric cancer in the late 1990s in Japan, and it has rapidly spread, surpassing conventional endoscopic mucosal resection (EMR) [5-7]. ESD has been developed as a new technique, and as en bloc endoscopic resection of large lesions and lesions with ulcer scar become possible [8]. Endoscopic treatment for digestive tract tumors has entered a new era.

ESD potentially includes problems such as technical difficulty, considerable time required to learn the skill, and frequent occurrence of complications such as hemorrhage and perforation [9-11], on the other hands, ESD enables higher rates of en block resection than EMR, and as a result, clearly reduces recurrence from residual tumors [12]. Instruments for ESD and related devices have been developed with improvements in, and stabilization of, its procedure, and the technique has spread gradually at many facilities.

In this report, we describe the indications of endoscopic treatment for early gastric cancer (EGC), technical details of ESD, diagnosis of the tumor extent during treatment, complications, and their management.

\footnotetext{
"Corresponding author.
}

\section{Indication of Endoscopic Therapy for Early Gastric Cancer}

In Japan, EGC is defined as a gastric carcinoma confined to the mucosa or submucosa, regardless of the presence or absence of regional lymph-node metastases. The outcome of surgical therapy for EGCs has been favorable: the 5-year survival rate reaches $90 \%-100 \%$ in Japan [13-16]. Endoscopic therapy may be considered as a treatment option for EGC because of its low invasiveness, but it can only be a valid alternative to surgery if the rate of complete cure is maintained at the same level as that of surgical therapy. Thus, the indications of endoscopic therapy for EGC are based on evaluating the possibility of complete cure after endoscopic treatment.

According to the "Gastric Cancer Treatment Guidelines” published by the Japanese Gastric Cancer Association in 2001, the indications for EMR of EGC are restricted to a non-ulcerated, differentiated-type mucosal carcinoma measuring less than $2 \mathrm{~cm}$ [17]. Basically, the lesion must have a negligible risk of lymph-node metastasis, and its size and location should not preclude the possibility of en bloc resection. Submucosal invasion, ulceration, and the undifferentiated type are important risk factors for lymph-node metastasis, as shown in large studies of surgically resected specimens [18-20]. Regarding the size and location of the lesions, the criterion is based on the recognition that en bloc resection is essential. Piecemeal resection impairs pathological judgment as to whether or not the lesion has been completely 
resected and increases the risk of residual cancer and local recurrence [7,12,21].

ESD has been developed to expand indications of endoscopic treatment for stomach cancer and to reliably resect large lesions, the en bloc resection of which has been difficult by conventional EMR using a snare. The development of this technique has made the limitation of the tumor size that that can be resected en bloc by EMR $(<2 \mathrm{~cm})$ meaningless, and the widening of indications of endoscopic treatment for stomach cancer is evaluated.

\subsection{Patients to Be Excluded from ESD}

ESD is a procedure that is less invasive than open surgery for stomach cancer, preserves the stomach function, and ensures a high quality of life. However, compared with EMR, ESD is associated with a higher incidence of procedural accidents, the severity of which is higher [12]. Early gastric cancer, which can be treated by ESD, has been detected more frequently in elderly patients suffering from various diseases due to the aging of the population. ESD is performed only if long-term survival is expected, and the patient's general condition is such that procedural accidents, if any, can be remedied by surgery. It should be avoided, if possible, to choose ESD because the patient's general condition is too poor for surgery.

\subsection{Technique of ESD}

\subsubsection{Selection and Preparation of the Endoscope}

ESD for early gastric cancers is carried out using a singlechannel upper gastrointestinal endoscope with a water-jet system ( GIF-Q260J; Olympus Optical Co., Tokyo, Japan) and a high-frequency generator with an automatically controlled system (ENDOCUT mode) (Erbotom ICC 200; ERBE Elektromedizin GmbH, Tubingen, Germany). For ESD of the stomach, the use of an upper gastrointestinal endoscope with a multi-bending function provided by 2 flexible parts (GIF-2TQ260M; Olympus Optical Co., Tokyo, Japan) is an option to treat lesions that are difficult to approach. An attachment-loaded balloon that facilitates access to difficult-to-approach sites is also commercially available.

ESD must be performed by attaching a transparent hood to the tip of the endoscope to open up the incised mucosa as a substitute for countertraction and also to help control the movements of the knife.

\subsubsection{Knife Selection}

Presently, many types of ESD knife are sold. A few representative knives are described (Figure 1).

Flush knife (DK2618N; Fujinon, Tokyo, Japan)

Since the author uses a flush knife as a basic knife, its characteristics and the method for its use are described in some detail.

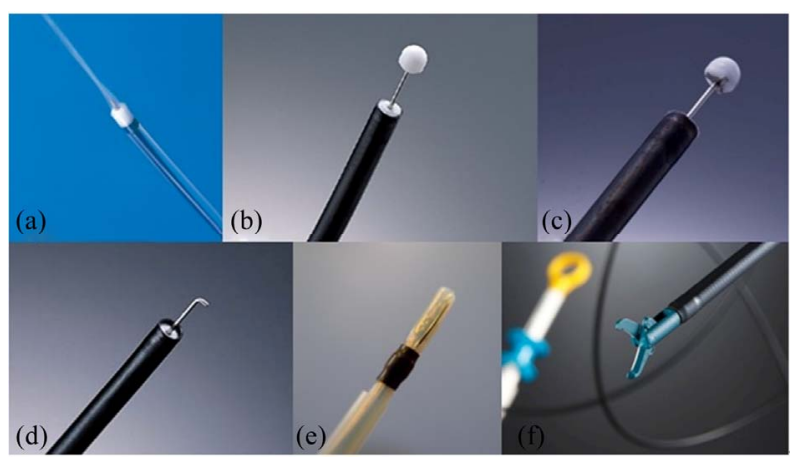

Figure 1. Various types of ESD knife. (a) Flush knife; (b) IT knife; (c) IT knife 2; (d) Hook knife; (e) Mucosectome; (f) SB knife.

It is a short needle knife with an irrigation function. Five types with different tip lengths ranging from $1-3$ $\mathrm{mm}$ at $0.5-\mathrm{mm}$ intervals are available. The $1.5-\mathrm{mm}$ type is suited for the colon and esophagus, and the $2.5-\mathrm{mm}$ type is suited for the stomach. Since adjustment of the tip length or direction is unnecessary, it can be set-up quickly and makes safe and easy manipulation possible using the sheath as a protector. The tip of the sheath can be used to push the muscle layer and lesion aside and be advanced into the narrow submucosal layer, and hard fibers can be dissected without difficulty. The greatest advantage is that washing of the surgical field or additional submucosal injection can be performed by flushing water through the knife without changing the instrument, resulting in marked improvements in the efficiency and safety of the procedure.

IT knife and IT knife 2 (KD-610L, DK-611L; Olympus, Tokyo, Japan)

The IT knife is an endoscopic instrument with a small ceramic ball attached as an insulator to the end of a needle knife to prevent perforation. The insulator at the tip contributes to the prevention of perforation. The tip of the knife is blunt, and cutting is performed using the blade on the side of the knife. Speedy cutting over a long distance is a characteristic of this instrument. In IT knife 2 , a new electrode installed at the tip of the knife has improved the ability to make transverse incisions.

Hook knife (KD-620LR; Olympus, Tokyo, Japan)

It is a needle knife with a tip perpendicularly bent at $1.4 \mathrm{~mm}$ from its end to hook mucosal or submucosal fibers and incise or dissect them. The risk of perforation can be reduced even when the knife must be advanced perpendicularly to the muscle layer and in patients with marked fibrosis as long as it is manipulated by pulling. However, as the direction of the knife must be adjusted, because the length that can be cut by one stroke of hooking is short, ESD using this knife alone takes time. It is frequently used for special purposes such as dissection of a markedly fibrosed submucosal layer. 
Mucosectome (DP-D2518; HOYA, Tokyo, Japan)

Mucosectome is a circumferentially insulated knife with a single-wire blade on one side of the tip. Since the entire circumference of the knife is covered by a plastic insulator, dissection can be performed safely by positioning the plastic part on the muscle layer. Since the tip is also an insulator, a long distance can be cut quickly, similarly to the IT knife, by applying the tip and swinging it sideways.

SB knife (MD-47703; Sumitomo Bakelite, Tokyo, Japan)

It is a scissors-like knife with electrodes placed on the inner sides of the blades and nonconductive coating of the external surface. By holding the knife and pulling it toward the surgeon, incision or dissection can be accomplished safely and easily. Since it can clip a vessel and coagulate blood, it also serves as hemostatic forceps.

The author also uses this knife for releasing membranous stenosis of the esophagus.

\section{Endoscopic Submucosal Dissection Procedure (Figure 2)}

\subsection{Diagnosis of Tumor Extent}

The advantage of ESD is that it enables the en masse resection of even large lesions, thereby allowing the accurate pathological evaluation of the resected lesions and avoidance of recurrence after piecemeal resection. Therefore, it is important to accurately determine the extent of gastric cancer invasion and resect the cancer.

However, in gastric cancer, it is sometimes difficult to determine the extent of tumor invasion as clearly as in iodine staining for detecting esophageal cancer. Therefore, the extent of gastric cancer invasion is usually determined comprehensively based on endoscopic findings and findings using indigo carmine dye spray (Figure 2(A)). If it is still difficult to determine the tumor extent, it is determined based on the findings of biopsy of the area around the lesion as well.

In the last several years, the usefulness of NBI magnifying observation not only in the qualitative diagnosis of gastric cancer but also in the diagnosis of the tumor extent has been reported [22-24].

For magnifying NBI-guided assessment of the stomach, it is necessary to analyze microvascular features and fine mucosal structures separately and make a diagnosis based on these findings. NBI magnifying observation is very useful in many patients (Figures $\mathbf{3}(\mathbf{A})$ and (B)). However, it is difficult to diagnose the tumor extent by NBI magnifying observation in some cancers, such as an undifferentiated cancer extending into the gland neck and covered with a normal mucosa and a gastritis-like type of differentiated adenocarcinoma (Figures 3(C) and (D)). Therefore, it is essential to make a comprehensive diag-

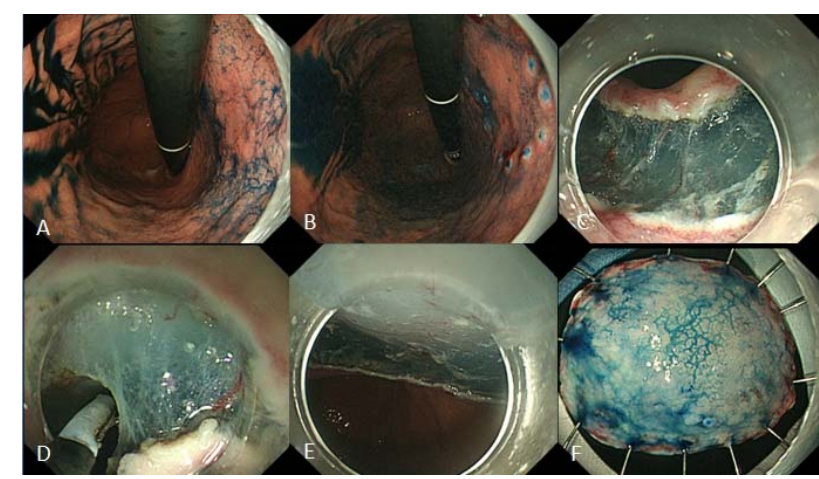

Figure 2. Typical example of ESD for early gastric cancer. (A) Indigo carmine was sprayed to clarify the border of the superficial depressed lesion; (B) Marking placement around the lesion; (C) Initial mucosal incision after submucosal injection at the distal margin of the lesion and submucosal dissection from distal end; (D) Mucosal incision after submucosal injection at the proximal margin of the lesion and submucosal dissection from the proximal end; (E) Mucosal defect after ESD; (F) Resected specimen.

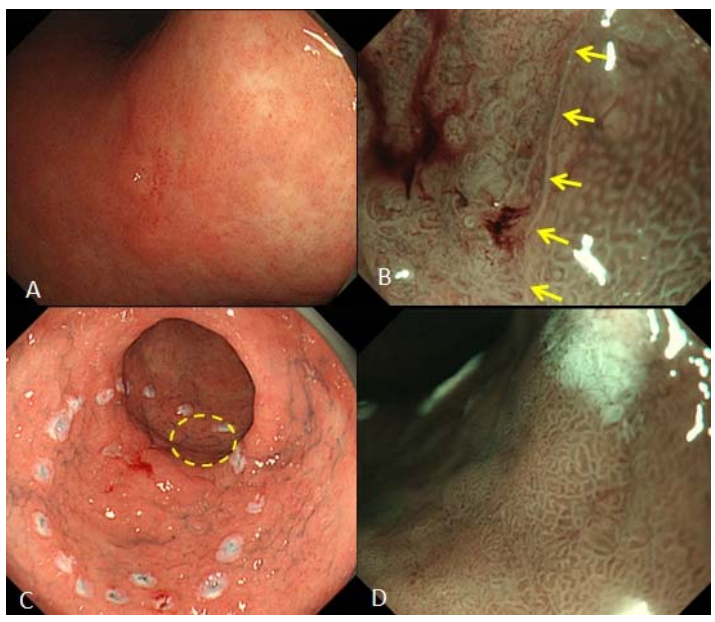

Figure 3. Examples of diagnosis of the tumor extent by NBI observation (A-B, Successful NBI diagnosis; C-D, Limitation of NBI diagnosis). (A) A 15-mm, depressed lesion was observed in the lesser curvature of the gastric body. It was very difficult to diagnose the lesion extent by conventional endoscopy; (B) NBI-magnifying observation facilitated identification of the tumor border based on the difference in the appearance of glandular structures and small blood vessels in the cancerous and non-cancerous areas; (C) A depressed lesion with fur was observed in the greater curvature of the gastric antrum. It was very difficult to diagnose the lesion extent by conventional endoscopy. After NBI magnifying observation with dye spraying, marking dots were made circumferentially sufficiently away from the lesion. The encircled area was judged as non-cancerous mucosa; (D) The encircled area in Figure $C$ observed by NBI magnifying endoscopy. No definite abnormal findings were observed, and the findings were interpreted as non-cancerous (gastritis). Histopathological examination of the specimen resected after ESD showed cancer progression in the same area, leading to the diagnosis of a positive horizontal margin. 
nosis based on findings including those of biopsies.

\subsubsection{Marking around the Lesion}

The safety margin from the end of the lesion should be at least $3 \mathrm{~mm}$ from the viewpoint of postoperative pathological examination.

Marking is made using the tip of the Flush knife. Dots are made circumferentially at even intervals $3 \mathrm{~mm}$ or more away from the lesion (Figure 2(B)). Points for marking are not to press the tip too hard against the mucosa and to shorten the duration of electricity application.

The soft coagulation mode (output, 40 - $50 \mathrm{~W}$ ) was used as the electronic current.

\subsubsection{Creating a Submucosal Fluid Cushion}

To secure clear vision and perform ESD safely under direct observation, submucosal injection should be performed frequently. Physiologic saline, Glyceol, sodium hyaluronate solution, etc., are used for submucosal injection in ESD. The viscosity of these solutions increases in this order, and a hard elevation is maintained for longer as a more viscous solution is used.

Indigo carmine may be added to facilitate the recognition of the submucosal layer, into which injection has been made, or to contrast the lesion against blood vessels and the muscle layer, or epinephrine may be added to prevent hemorrhage. Figure 4 shows the difference in the appearance of the gastric submucosa after the submucosal injection of a mixture of sodium hyaluronate solution and indigo carmine or sodium hyaluronate solution alone.

Since the submucosal injection of sodium hyaluronate solution into the muscle layer or deeper areas makes subsequent procedures difficult, it is recommended to inject physiologic saline first and then inject sodium hyaluronate solution after confirmation of its entry into the submucosal layer.

\subsubsection{Incising the Mucosa outside the Lesion}

After lifting the lesion, a mucosal incision was made with a Flush knife. The knife was gently pressed onto the mucosa to produce a cutting effect using the ENDOCUT mode with effect 3 (output, 80 - $120 \mathrm{~W}$ ).

In ESD of the stomach, incision and dissection from the oral side may cause the falling of the lesion into the duodenum and makes subsequent manipulations difficult, so incision should be made first on the anal side of the lesion. Another point of caution is that satisfactory elevation cannot be achieved in large lesions, which take time to resect, if a circumferential incision is made first, because the injection fluid leaks through the incision even with additional submucosal injections. It is important to make a partial rather than circumferential incision first, immediately dissect the submucosal layer, and repeat this

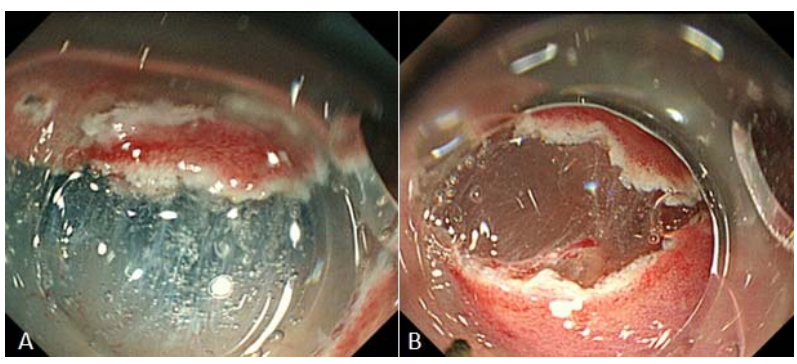

Figure 4. The difference in the appearance of the gastric submucosa after the submucosal injection of a mixture of sodium hyaluronate solution and indigo carmine or sodium hyaluronate solution alone. (A) With indigo carmine. It was easy to visually distinguish the submucosal layer stained blue with indigo carmine and the white muscular layer; (B) Without indigo carmine. Compared with Figure (A), the border between the submucosal and muscular layers was ill-defined.

process.

\subsubsection{Dissecting the Submucosal Layer Beneath the Lesion}

After partial incision, dissection of the submucosal layer is started immediately, with the Flush knife used to make the first incision (forced coagulation, 35 - 45 W). Dissection is initiated by applying the knife to the inside (on the tumor side) of the incision margin and advanced along the incision margin. As dissection is advanced, the incised wound opens gradually, and the submucosal layer becomes easier to approach. As dissection is advanced further, a space sufficient to insert the entire tip hood is created in the submucosal layer. By inserting a transparent tip hood, not only is good visibility of the submucosal layer achieved, but also dissection is facilitated further, because appropriate traction is applied to fibers. This manipulation is a very important point when using instruments to be mounted on the tip such as the Flush knife. To conduct safe and effective ESD, it is important to repeat submucosal injection, incision, and dissection with a favorable balance so that the tumor is released evenly.

In patients showing marked fibrosis making dissection of the submucosal layer difficult and those in whom respiratory movements and the pulse interfere with safe the execution of dissection, safe dissection can be achieved by applying electricity after pinpoint pulling of the submucosal fibers to be dissected into the tip hood using a hook knife (KD-620LR; Olympus) or scissors-like SB knife (MD-47703; Sumitomo Bakelite, Tokyo, Japan). However, since only a small number of fibers can be held at a time, the treatment is time-consuming.

On the other hand, for dissection of the submucosal layer in a large lesion, the operation time may be shortened by dissecting a wide area at one time using a knife such as the mucosectome (DP-D2518; HOYA). 


\subsection{Complications and Measures to Control Them}

Major complications associated with ESD of include perforation and bleeding (Figure 5). First, the incidence of perforation has been reported to be about $1.2 \%-6.1 \%$ even after ESD of the stomach [9-11], which is technically the most established and widely performed, and is higher than that of EMR. Particularly, the risk of perforation is high, and caution is needed, in patients with large lesions, lesions of the upper body of the stomach, and those with ulcer scars. Pneumoperitoneum is caused by air leak from the site of perforation. If the air leak is small, it poses no problem, but a large air leak may cause exacerbation of the respiratory state or neurogenic shock. If perforation has occurred or is suspected, the abdomen of the patient must be palpated frequently to evaluate pneumoperitoneum, and the gas must be released through peritoneocentesis if pneumoperitoneum is severe or changes are observed in vital signs. Peritoneocentesis must be performed after confirming that the intestine is not located at the puncture site under ultrasound guidance as much as possible.

Recently, air leak has ceased to be a serious problem due to insufflation of $\mathrm{CO}_{2}$ during ESD. Since $\mathrm{CO}_{2}$ is absorbed more rapidly than air, it reduces the patient's discomfort due to an increase in gas in the intestine associated with a prolonged procedure, and if it should leak into the abdominal cavity due to perforation, it is absorbed relatively quickly. However, attention to $\mathrm{CO}_{2}$ narcosis is necessary when it is used in COPD patients.

Conventionally, emergency surgery was considered necessary to treat perforation during endoscopic treatment. However, there is no food residue in the stomach during gastric ESD, and no bacteria can usually inhabit the stomach. Therefore, even if the stomach is perforated, serious bacterial peritonitis does not occur if the perforation site is promptly closed by clipping, necessary treatment of pneumoperitoneum is made, eating and drinking are avoided, decompression is made by gastric intubation through the nasal route, and antibiotics are administered, and the condition is often resolved in a few days only after mild localized peritonitis due to the leakage of gastric juice.

Although perforation due to ESD can be managed reasonably by conservative treatments as those mentioned above in most patients, conservative treatments should not be adhered to, and, always with the possibility of surgical treatments in mind, cooperation with surgeons must be secured.

Regarding bleeding, some intraoperative bleeding is almost unavoidable. Since bleeding makes securing of the visibility of the surgical field difficult, it is important first to prevent bleeding. Preventive cauterization of small vessels observed during incision and dissection

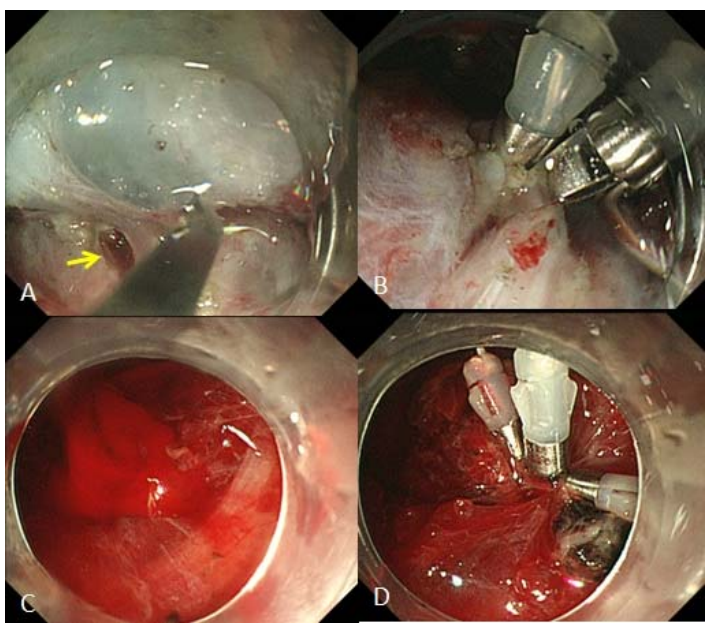

Figure 5. Complications and their management. (A) During submucosal dissection, a pinhole perforation was noted; (B) The perforation was closed with clips; (C) Bleeding due to vascular injury during submucosal dissection; (D) Since hemostasis was difficult using hemostatic forceps, it was performed using hemoclips.

with the knife being used before the occurrence of bleeding is important. If bleeding is from a relatively large vessel or difficult to stop with a knife, hemostatic forceps should be used. It is the most important to promptly determine the site of bleeding and achieve hemostasis by employing as few manipulations as possible. If the visibility is impaired due to the pooling of blood by severe bleeding, a change in the body position may be effective. In addition, the use of an endoscope with a water jet function is considered effective to shorten the time until hemostasis and more efficiently secure the visibility by irrigation. Desperate attempts to stop bleeding may cause damage to the mucosal side of the lesion or lamina propria mucosae and increase the risk of late perforation.

\subsection{Handling of Resected Specimens}

According to the $3^{\text {rd }}$ English Edition of the Japanese Classification of Gastric Carcinoma [25], specimens obtained by endoscopic or laparoscopic mucosal resection should be handled in the following manner: the specimen should be spread out, pinned on a flat cork, and fixed in formalin solution. The size of the specimen, the size and shape of the tumor, and the margins should be recorded on a schematic diagram. The proximal cut end should be indicated by an arrow, if possible. Fixed materials should be sectioned serially at $2 \mathrm{~mm}$ intervals parallel to a line that includes the closest resection margin of the specimen.

\subsection{Histological Examination}

The histological type and the size of the largest dimension of the tumor, the presence or absence of ulceration 
(UL), lymphatic invasion (ly) and venous invasion (v) should be recorded. The depth of invasion (M, SM1, SM2) are determined and recorded only when the Vertical margin (VM) is negative (SM1, submucosal invasion $<0.5 \mathrm{~mm}$; SM2, invasion $\geqq 0.5 \mathrm{~mm}$ ).

The lateral margin (LM) should be assessed, and if LM is negative, the length (millimeter) of the free margin or the number of normal tubules in the margin are recorded. Tumor extent, together with depth of invasion, should be recorded on a schematic diagram.

\section{Summary}

We describe the indications, technical details, and complications of ESD for early gastric cancer.

To perform ESD safely and reliably, not only the techniques of incision and submucosal dissection, but also the management of intraoperative bleeding and perforation is important.

Although problems such as the difficulty in mastering the procedure and accidents remain to be overcome, ESD is expected to spread widely not only in Japan but also abroad as a mildly invasive treatment for early gastric cancer.

\section{REFERENCES}

[1] M. Tada, A. Murakami, M. Karita, H. Yanai and K. Okita, "Endoscopic Resection of Early Gastric Cancer," Endoscopy, Vol. 25, No. 7, 1993, pp. 445-450. doi:10.1055/s-2007-1010365

[2] A. Torii, M. Sakai, T. Kajiyama, et al., "Endoscopic Aspiration Mucosectomy as Curative Endoscopic Surgery; Analysis of 24 Cases of Early Gastric Cancer," Gastrointestinal Endoscopy, Vol. 42, No. 5, 1995, pp. 475-479. doi:10.1016/S0016-5107(95)70053-6

[3] H. Inoue, M. Endo, K. Takeshita, et al., "A New Simplified Technique of Endoscopic Esophageal Mucosal Resection Using a Cap-Fitted Panendoscope (EMRC)," Surgical Endoscopy, Vol. 6, No. 5, 1992, pp. 264-265. doi:10.1007/BF02498820

[4] H. Inoue, K. Takeshita, H. Hori, et al., "Endoscopic Mucosal Resection with a Cap-Fitted Panendoscope for Esophagus, Stomach, and Colon Mucosal Lesions,” Gastrointestinal Endoscopy, Vol. 39, No. 1, 1993, pp. 58-62. doi:10.1016/S0016-5107(93)70012-7

[5] H. Yamamoto, T. Yube, N. Isoda, et al., "A Novel Method of Endoscopic Mucosal Resection Using Sodium Hyaluronate," Gastrointestinal Endoscopy, Vol. 50, No. 2, 1999, pp. 251-256. doi:10.1016/S0016-5107(99)70234-8

[6] T. Gotoda, H. Kondo, H. Ono, et al., “A New Endoscopic Mucosal Resection Procedure Using an Insulation-Tipped Electrosurgical Knife for Rectal Flat Lesions: Report of Two Cases," Gastrointestinal Endoscopy, Vol. 50, No. 4, 1999, pp. 560-563. doi:10.1016/S0016-5107(99)70084-2

[7] H. Ono, H. Kondo and T. Gotodav, "Endoscopic Mucosal Resection for Treatment of Early Gastric Cancer,” Gut,
Vol. 48, No. 2, 2001, pp. 225-229. doi:10.1136/gut.48.2.225

[8] Y. Takeuchi, N. Uedo, H. Iishi, et al., "Endoscopic Submucosal Dissection with Insulated-Tip Knife for Large Mucosal Early Gastric Cancer: A Feasibility Study (with videos)," Gastrointestinal Endoscopy, Vol. 66, No. 1, 2007, pp. 186-193. doi:10.1016/j.gie.2007.03.1059

[9] I. K. Chung, J. H. Lee, S. H. Lee, et al., "Therapeutic Outcomes in 1000 Cases of Endoscopic Submucosal Dissection for Early Gastric Neoplasms: Korean ESD Study Group Multicenter Study,” Gastrointestinal Endoscopy, Vol. 69, No. 7, 2009, pp. 1228-1235. doi:10.1016/j.gie.2008.09.027

[10] T. Ohta, R. Ishihara, N. Uedo, et al., "Factors Predicting Perforation during Endoscopic Submucosal Dissection for Gastric Cancer,” Gastrointestinal Endoscopy, Vol. 75, No. 6, 2012, pp. 1159-1165. doi:10.1016/j.gie.2012.02.015

[11] A. Imagawa, H. Okada, Y. Kawahara, et al., "Endoscopic Submucosal Dissection for Early Gastric Cancer: Results and Degrees of Technical Difficulty as Well as Success," Endoscopy, Vol. 38, No. 10, 2006, pp. 987-990. doi:10.1055/s-2006-944716

[12] J. Lian, S. Chen, Y. Zhang and F. Qiu, “A Meta-Analysis of Endoscopic Submucosal Dissection and EMR for Early Gastric Cancer," Gastrointestinal Endoscopy, Vol. 76, No. 4, 2012, pp. 763-770. doi:10.1016/j.gie.2012.06.014

[13] H. Onodera, A. Tokunaga, T. Yoshiyuki, et al., "Surgical Outcome of 483 Patients with Early Gastric Cancer: Prognosis, Postoperative Morbidity and Mortality, and Gastric Remnant Cancer,” Hepatogastroenterology, Vol. 51, No. 55, 2004, pp. 82-85.

[14] K. Inoue, T. Tobe, N. Kan, et al., "Problems in the Definition and Treatment of Early Gastric Cancer," British Journal of Surgery, Vol. 78, No. 7, 1991, pp. 818-821. doi:10.1002/bjs.1800780717

[15] Y. Maehara, H. Orita, T. Okuyama, et al., "Predictors of Lymph Node Metastasis in Early Gastric Cancer,” British Journal of Surgery, Vol. 79, No. 3, 1992, pp. 245-247. doi:10.1002/bjs. 1800790320

[16] Y. Seto, H. Nagawa and T. Muto, "Impact of Lymph Node Metastasis on Survival with Early Gastric Cancer," World Journal of Surgery, Vol. 21, No. 2, 1997, pp. 186-189. doi:10.1007/s002689900213

[17] Japanese Gastric Cancer Association, "Guidelines for the Treatment of Gastric Cancer," Kanehara \& Co., Ltd., Tokyo, 2001.

[18] T. Sano, O. Kobori and T. Muto, "Lymph Node Metastasis from Early Gastric Cancer: Endoscopic Resection of Tumour,” British Journal of Surgery, Vol. 79, No. 3, 1992, pp. 241-244. doi:10.1002/bjs.1800790319

[19] T. Yamao, K. Shirao, H. Ono, et al., "Risk Factors for Lymph Node Metastasis from Intramucosal Gastric Carcinoma,” Cancer, Vol. 77, No. 4, 1996, pp. 602-606. doi:10.1002/(SICI)1097-0142(19960215)77:4<602::AIDCNCR3>3.0.CO;2-I

[20] T. Namieno, K. Koito, T. Higashi, et al., "Assessing the Suitability of Gastric Carcinoma for Limited Resection: Histologic Differentiation of Endoscopic Biopsy,” World 
Journal of Surgery, Vol. 22, No. 8, 1998, pp. 865-868. doi:10.1007/s002689900483

[21] M. Matsushita, K. Hajiro, K. Okazaki and H. Takakuwa, "Endoscopic Mucosal Resection of Gastric Tumors Located in the Lesser Curvature of the Upper Third of the Stomach,” Gastrointestinal Endoscopy, Vol. 45, No. 6, 1997, pp. 512-515. doi:10.1016/S0016-5107(97)70183-4

[22] M. Kaise, M. Kato, M. Urashima, et al., "Magnifying Endoscopy Combined with Narrow-Band Imaging for Differential Diagnosis of Superficial Depressed Gastric Lesions,” Endoscopy, Vol. 41, No. 4, 2009, pp. 310-315. doi:10.1055/s-0028-1119639

[23] K. Yao, T. Matsui and A. Iwashita, "Clinical Application of Magnification Endoscopy with NBI for Diagnosis of Early Gastric Cancer,” Nippon Shokakibyo Gakkai Zasshi, Vol. 104, 2007, pp. 782-789

[24] K. Nonaka, M. Namoto, H. Kitada, et al., "Usefulness of the DL in ME with NBI for Determining the Expanded Area of Early-Stage Differentiated Gastric Carcinoma," World Journal of Gastrointestinal Endoscopy, Vol. 4, 2012, pp. 362-367. doi:10.4253/wjge.v4.i8.362

[25] T. Sano and Y. Kodera, "Japanese Classification of Gastric Carcinoma: 3rd English Edition,” Gastric Cancer, Vol. 14, No. 2, 2011, pp. 101-112. doi:10.1007/s10120-011-0041-5 